\title{
BMJ
}

\section{Effect on falls of providing single lens distance vision glasses to multifocal glasses wearers: VISIBLE randomised controlled trial}

\author{
Mark J Haran, rehabilitation medicine physician, ${ }^{1}$ Ian D Cameron, professor of rehabilitation medicine, ${ }^{2}$ \\ Rebecca Q Ivers, associate professor of injury prevention, ${ }^{3,4}$ Judy M Simpson, professor of biostatistics ${ }^{4}$ \\ Bonsan B Lee, rehabilitation medicine physician, ${ }^{5,6,7}$ Michael Tanzer, optometrist, ${ }^{7}$ Mamta Porwal, research \\ assistant,, Marcella M S Kwan, research assistant, ${ }^{7}$ Connie Severino, research assistant,, Stephen R Lord, \\ senior principal research fellow ${ }^{6,7}$
}

1Department of Aged Care and Rehabilitation, Royal North Shore Hospital, St Leonards, Sydney,

Australia

${ }^{2}$ Rehabilitation Studies Unit, Sydney Medical School, University of Sydney, Sydney

${ }^{3}$ Injury Division, George Institute for International Health, University of Sydney

${ }^{4}$ Sydney School of Public Health, University of Sydney

${ }^{5}$ Department of Rehabilitation, Prince of Wales Hospital, Sydney

${ }^{6}$ School of Public Health and

Community Medicine, University of New South Wales (UNSW), Sydney

${ }^{7}$ Prince of Wales Medical Research Institute, UNSW, Randwick, Sydney, NSW 2031, Australia

Correspondence to: $\mathrm{S}$ Lord s.lord@powmri.edu.au

Cite this as: $B M J$ 2010;340:c2265 doi:10.1136/bmj.c2265

\section{ABSTRACT}

Objective To determine whether the provision of single lens distance glasses to older wearers of multifocal glasses reduces falls.

Design Parallel randomised controlled trial stratified by recruitment site and source of referral, with 13 months' follow-up and outcome assessors blinded to group allocation.

Setting Community recruitment and treatment room assessments in Sydney and Illawarra regions of NSW, Australia.

Participants 606 regular wearers of multifocal glasses (mean age 80 (SD 7) years). Inclusion criteria included increased risk of falls (fall in previous year or timed up and go test $>15$ seconds) and outdoor use of multifocal glasses at least three times a week.

Interventions Provision of single lens distance glasses with recommendations for wearing them for walking and outdoor activities compared with usual care.

Main outcome measures Number of falls and injuries resulting from falls during follow-up.

Results Single lens glasses were provided to 275 (90\%) of the 305 intervention group participants within two months; 162 (54\%) of the intervention group reported satisfactory use of distance glasses for walking and outdoor activities for at least 7/12 months after dispensing. In the 299 intervention and 298 control participants available to follow-up, the intervention resulted in an $8 \%$ reduction in falls (incidence rate ratio $0.92,95 \%$ confidence interval 0.73 to 1.16 ). Pre-planned sub-group analyses showed that the intervention was effective in significantly reducing all falls (incidence rate ratio $0.60,0.42$ to 0.87 ), outside falls, and injurious falls in people who regularly took part in outside activities. A significant increase in outside falls occurred in people in the intervention group who took part in little outside activity.

Conclusions With appropriate counselling, provision of single lens glasses for older wearers of multifocal glasses who take part in regular outdoor activities is an effective falls prevention strategy. The intervention may be harmful, however, in multifocal glasses wearers with low levels of outdoor activity.

Trial registration Clinical trials NCT00350855.

\section{INTRODUCTION}

Presbyopia is the most prevalent form of visual impairment in older people. ${ }^{1}$ To correct for this condition, older people are either prescribed separate single lens glasses for distant and near vision or, for convenience, a single pair of multifocal (bifocal, trifocal, or progressive lens) glasses. Multifocal glasses have benefits for tasks that require changes in focal length, including everyday tasks of driving, shopping, and cooking. However, multifocal glasses also have disadvantages. The most common type of multifocal glasses-bifocal glasses - have optical defects, such as prismatic jump at the top of the reading segment, which causes an apparent displacement of fixed objects. ${ }^{12}$ The lower lenses of all types of multifocal glasses blur distant objects in the lower visual field, and this factor in particular may represent an important problem for older people. ${ }^{23}$

Multifocal glasses have been shown to impair distant depth perception and contrast sensitivity. ${ }^{4}$ Three recent studies in older people have found that multifocal glasses impair step negotiation and accuracy of foot placement when stepping on to a raised surface or negotiating an obstacle course. ${ }^{5-7}$ Other studies have reported that wearing multifocal glasses increases the risk of trip incidents and falls in older people. ${ }^{489}$ In particular, wearers of multifocal glasses have a high risk of falls when outside their homes and when walking up or down stairs. ${ }^{4}$

Two previous randomised controlled trials have evaluated the efficacy of visual interventions that have provided updated glasses as the primary intervention. The first, involving 1090 participants aged 70 years and over, found that an intervention comprising a referral to an eye care provider resulted in only a $4 \%$ reduction in the rate of falls over one year. ${ }^{10}$ The 


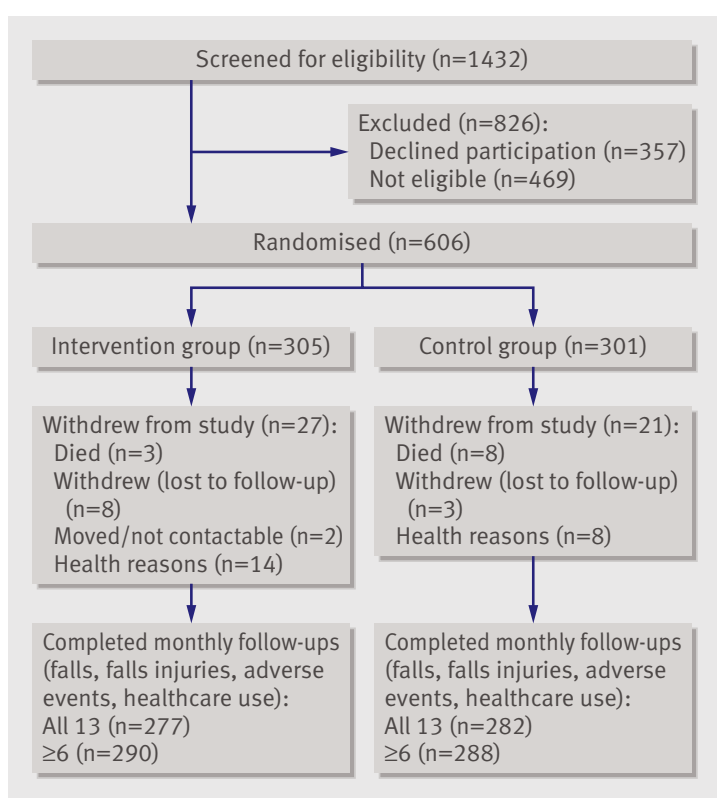

Flow of participants through study

second found that an intervention involving comprehensive assessment of vision and subsequent treatment for identified eye problems significantly increased the rate of falls by more than $50 \%$ in the intervention group. ${ }^{11}$ The authors concluded that the intervention group participants might have needed more time to adapt to their updated prescriptions or new glasses or may have adopted more risk taking activities and thus increased the exposure to falls after their improvement in vision. Neither intervention involving updating glasses aimed to restrict the use of multifocal glasses; in not doing so, they may have left an important risk factor unaltered.

The primary aim of the Visual Intervention Strategy Incorporating Bifocal and Long-distance Eyewear (VISIBLE) randomised controlled trial was to determine whether the provision of single lens distance glasses to older wearers of multifocal glasses, with counselling about optimal use when taking part in hazardous activities, could reduce rates of falling.

\section{METHODS}

\section{Participants}

We recruited participants for the trial between May 2005 and June 2007. Participants were eligible if they were community dwelling and at a relatively high risk of falls, defined as either age 80 years and over or aged 65 years and over and having had either a fall in the previous 12 months or a timed up and go test score of at least 15 seconds; used bifocal, trifocal, or progressive lens glasses at least three times a week when walking outdoors; had been reviewed by an optometrist or ophthalmologist in the previous 24 months; did not currently use single lens distance glasses; and indicated that they were at least "quite or very confident" that they could comply with the study recommendations. The sample size calculation, based on a $23 \%$ reduction in the rate of falls in the intervention group with $80 \%$ power and a significance level of $5 \%$, indicated that we needed 580 participants (290 per group). Full details of the inclusion criteria and methods are reported in the VISIBLE study protocol paper. ${ }^{12}$

\section{Initial assessment}

The figure shows the flow of participants in the trial. After the study aims were explained, participants who indicated that they could confidently manage to wear two pairs of glasses gave informed consent and had subsequent assessments of vision and physical functioning before randomisation into the study.

\section{Randomisation procedure}

After the initial assessment, research personnel (MK and MP) randomised participants to the intervention or control group, with stratification by recruitment site and source of referral (hospital $v$ community). Each stratum was randomly allocated in permuted blocks of 10 generated externally (by JS) by using sequentially numbered opaque envelopes containing group assignment.

\section{Intervention}

Participants in the intervention group had an examination by an optometrist, which included measurement of objective refraction. They were then prescribed a pair of single lens distance glasses. For intervention group participants who had a significant change in distance correction since their most recent prescription, updated multifocal lenses were provided. The distance component of the updated multifocal correction was then used for the prescription of new single lens distance glasses, making the transition between the two types of glasses easier. For intervention group participants who did not need an update of multifocal lens prescription, the correction for the single lens glasses was matched with the prescription of the distance segment of their current multifocal glasses. ${ }^{12}$ To reduce outdoor glare, participants were encouraged to accept transition lenses that become darker in sunlight for their new distance glasses or, if this was unacceptable, a tint of less than $30 \%$ or a graduated tint.

Participants received their glasses at a second visit. An optometrist demonstrated how multifocal glasses can impair visual abilities needed for detecting obstacles and judging depth. ${ }^{412}$ Participants were also shown photographs of hazardous street and step scenes with and without simulated lower field blur to reinforce why multifocal glasses may increase the risk of falls. ${ }^{12}$

The optometrist then instructed participants to use their new single lens glasses for most walking and standing activities, and in particular when taking part in the following activities: walking up or down stairs outside the home, walking in the street and at shopping centres, walking or standing in other people's homes and unfamiliar buildings, negotiating rough or uneven ground, and alighting from public transport. Use of multifocal glasses was not discouraged for seated 
tasks that need frequent changes in focal length such as driving and standing and walking tasks that require changes in focal depth where risk is minimal (such as cooking and walking in the home and selecting items in the supermarket). Intervention group participants were also provided with a booklet and reminder cards (with instructions on appropriate use of glasses) and a glasses cord or cloth glasses holder that could be worn around the neck or placed in the participant's pocket to aid in the swapping of glasses.

Participants in the control group had the same optometrist's examination as those in the intervention group. Participants who needed a prescription change were provided with updated multifocal lenses at a second visit without specific advice on the use of their glasses. ${ }^{12}$ No other fall prevention interventions, including strength and balance training, were offered to either the intervention or control participants.

\section{Outcomes}

The primary outcome measure was the number of falls in the 13 month follow-up period after randomisation (an average of 12 months' further follow-up after the receipt of glasses). We defined falls as "unintentionally coming to the ground or some lower level and other than as a consequence of sustaining a violent blow, loss of consciousness, sudden onset of paralysis as in stroke or an epileptic seizure" 13 and assessed them with monthly calendars and follow-up telephone calls as required. Injurious falls were those that resulted in fractures, dislocations, and organ and soft tissue trauma. Research personnel who received falls calendars, made follow-up phone calls, and entered data were blinded to group allocation.

Secondary outcome measures included physical activity levels, fear of falling (falls efficacy), and quality of life assessed at baseline and 12 months. These measures were included to determine whether, as a result of fewer falls, the intervention had beneficial effects on an older person's abilities and quality of life. Physical activity levels were self reported using the Adelaide activities profile. ${ }^{14}$ Physical and mental health were assessed with the SF-12. ${ }^{15}$ Falls efficacy was assessed with the falls efficacy scale-international. ${ }^{16}$

\section{Adherence and adverse events}

We measured participant reported adherence monthly in the intervention group (in months two to 13). The primary measure was the number of months in which adherence to the recommendation on wearing single lens distance glasses was followed most or all of the time when walking in the street or in shopping centres. Adverse effects such as non-falls injuries and falls occurring as a result of switching from single lens to multifocal glasses or vice versa, as well as healthcare resource use, were recorded on falls calendars.

\section{Statistical analysis}

The primary analyses were by intention to treat. We analysed the number of falls per person by using negative binomial regression to estimate the difference in rates of falls between the two groups. We did three pre-planned subgroup analyses using a test for statistical interaction. ${ }^{17} \mathrm{We}$ assessed whether the effect size of the intervention differed according to baseline physiological falls risk $(<1 \quad v \geq 1),{ }^{18}$ number of participant reported falls in the previous year (0-1 $v \geq 2)$, and baseline physical activity levels (using above or below median summed scores for the nine Adelaide activities profile outdoor activity items 8-11 and 19-21: household shopping, personal shopping, light gardening, heavy gardening, social activities, attending religious services/meetings, outdoor activities, sporting/recreational activities, and walking outside). ${ }^{14}$ In a final pre-planned analysis we assessed the effect of the intervention on the site of fall by separately analysing the effect of the intervention on participants' falls beyond their own home, including their own yard, as the outcome of interest. We used analysis of covariance with adjustment for baseline scores to assess the effect of group allocation on the continuously scored

Table 1 |Characteristics of participants in intervention and control groups at entry to study. Values are numbers (percentages) unless stated otherwise

\begin{tabular}{lcc} 
& $\begin{array}{c}\text { Intervention } \\
(\mathrm{n}=305)\end{array}$ & $\begin{array}{c}\text { Control } \\
(\mathrm{n}=301)\end{array}$ \\
\hline Demographic factors & $79.4(6.4)$ & $80.3(6.8)$ \\
\hline Mean (SD) age (years) & $119(39)$ & $94(31)$ \\
\hline Men
\end{tabular}

Multifocal lens type

\begin{tabular}{lcc}
\hline Bifocal & $192(63)$ & $173(57)$ \\
\hline Progressive & $66(22)$ & $79(26)$ \\
\hline Trifocal & $26(9)$ & $33(11)$ \\
\hline Two or more types & $20(7)$ & $14(5)$
\end{tabular}

Two or more types

Medical conditions

Mean (SD) mini mental state $\quad 28.5(1.5) \quad 28.6(1.4)$ examination score

Two or more falls in previous year $117(38) \quad 106(35)$

Stroke $22(7) \quad 24(8)$

Parkinson's disease 7 (2) $3(1)$

Heart disease $74(24) \quad 66(22)$

\begin{tabular}{lcc}
\hline Arthritis & $169(55)$ & $173(57)$
\end{tabular}

Diabetes

$56(18)$

$52(17)$

\section{Drug use}

\begin{tabular}{|c|c|c|}
\hline Four or more drugs & $188(62)$ & $208(69)$ \\
\hline Psychotropic drugs & $62(20)$ & $68(23)$ \\
\hline \multicolumn{3}{|l|}{ Vision and physical function } \\
\hline Mean (SD) visual acuity (MAR) & $1.40(0.54)$ & $1.54(0.98)$ \\
\hline $\begin{array}{l}\text { Mean (SD) edge contrast sensitivity } \\
\text { (dB) }\end{array}$ & $20.8(2.0)$ & $20.6(2.2)$ \\
\hline $\begin{array}{l}\text { Mean (SD) depth perception (cm } \\
\text { error)* }\end{array}$ & $3.1(3.4)$ & $3.3(4.5)$ \\
\hline $\begin{array}{l}\text { Mean (SD) sit to stand five times test } \\
\text { (s) }\end{array}$ & $16.4(9.6)$ & $15.3(2.0)$ \\
\hline Mean (SD) timed up and go test (s) & $12.8(6.7)$ & $14.8(8.3)$ \\
\hline Mean (SD) PPA fall risk score & $0.69(1.09)$ & $0.78(1.12)$ \\
\hline
\end{tabular}

MAR=minimum angle resolvable in minutes or arc; $P P A=$ physiological profile assessment.

*Error in aligning rods in Howard-Dohlman test. 
secondary outcome measures. We used the SPSS and Stata computer programs for all analyses.

\section{RESULTS}

\section{Baseline characteristics}

Table 1 shows the baseline demographic, cognitive, health, activity, and physical performance characteristics of the 606 participants. The groups were similar, although the intervention group $(n=305)$ contained more men and performed better in the timed up and go test.

\section{Intervention adherence and subject retention}

The median time to the second glasses dispensing visit was 28 (interquartile range 19-43) days for the intervention group and 29 (14-53) days for the control group. Twenty-seven (9\%) intervention group participants and $30(10 \%)$ control group participants were provided with updated multifocal glasses at these visits.

Two hundred and seventy five (90\%) intervention group participants received their single lens glasses within two months of randomisation. Nine intervention participants did not receive single lens glasses, two owing to diagnosed eye disease, one owing to dementia, and six owing to logistical difficulties. Thirty-five participants received additional counselling and support including follow-up telephone calls $(\mathrm{n}=24)$, replacement glasses $(\mathrm{n}=8)$, additional cords, glasses cases, reminder cards or "glasses use" information leaflets $(\mathrm{n}=8)$, and counselling sessions with optometrists $(\mathrm{n}=3)$.

The median number of months that the intervention participants reported satisfactory adherence with the recommendations for wearing single-lens glasses when walking in the street or in shopping centres was 7 (2-11) months. Ninety-six (32\%) of 299 participants adhered for 0-3 months, 41 (14\%) for 4-6 months, 40 (13\%) for 7-9 months, and $122(41 \%)$ for 10-12 months.
Two control group participants purchased distance glasses during the follow-up period.

By the end of the 13 month intervention period, 47 participants had withdrawn from the trial (28 from the intervention group and 19 from the control group), giving completion rates of $90 \%$ and $94 \%$. The figure shows the reasons for non-completion. The five deaths were unrelated to the intervention.

\section{Effect of intervention on falls}

Table 2 shows rates of falls and the number and proportion of intervention and control group participants who reported falls, falls outside the home, and injurious falls within the 13 month trial period; 299 intervention and 298 control participants completed one or more falls calendars. Blinding was maintained well throughout the study, although occasionally participants inadvertently revealed their group status (that is, mentioned new glasses) when research personnel made follow-up calls as part of the falls surveillance. In the 597 participants who completed calendars, fall rates did not differ significantly between the groups. In subgroup analysis, we found no differences in fall rates by baseline physiological falls risk $(\mathrm{P}=0.31)$ or number of reported falls in the previous year $(\mathrm{P}=0.19)$.

Significant interactions were evident between the intervention and self reported baseline outdoor activity levels (assessed with the Adelaide activities profile) for the outcomes of all falls, falls outside the home, and injurious falls $(\mathrm{P}<0.001)$. In more active participants with Adelaide activities profile subtotals for outdoor activities above the median of 15 , intervention group participants had significantly lower rates of all falls, falls outside the home, and injurious falls. In this subgroup, the numbers needed to treat to prevent one fall, one outdoor fall, and one injurious fall within 12 months were $1.1,1.9$, and 2.6. In contrast, in those who had Adelaide activities profile outdoor subtotal

Table 2| Falls, outside falls, and injurious falls in 13 month trial period

\begin{tabular}{|c|c|c|c|c|c|c|c|c|c|}
\hline & \multicolumn{4}{|c|}{ Intervention } & \multicolumn{4}{|c|}{ Control } & \multirow{2}{*}{$\begin{array}{c}\text { Incidence rate ratio } \\
(95 \% \mathrm{Cl})\end{array}$} \\
\hline & No & Fallers (\%)* & Total falls & Rate (SD)† & No & Fallers (\%)* & Total falls & Rate (SD)† & \\
\hline \multicolumn{10}{|l|}{ All falls } \\
\hline Overall & 299 & $170(57)$ & 461 & $1.54(2.40)$ & 298 & $175(59)$ & 496 & $1.66(3.04)$ & $0.92(0.73$ to 1.16$)$ \\
\hline Low AAP level & 151 & $93(62)$ & 274 & $1.81(2.75)$ & 185 & $107(58)$ & 252 & $1.36(1.68)$ & $1.29(0.95$ to 1.75$)$ \\
\hline High AAP level & 148 & $77(52)$ & 187 & $1.26(1.96)$ & 113 & $68(60)$ & 244 & $2.16(4.41)$ & $0.60(0.42$ to 0.87$)$ \\
\hline \multicolumn{10}{|l|}{ Outside falls } \\
\hline Overall & 299 & $139(46)$ & 197 & $0.83(1.19)$ & 298 & $124(42)$ & 192 & $0.85(1.57)$ & $1.00(0.78$ to 1.28$)$ \\
\hline LowAAP level $(n=342)$ & 151 & $77(51)$ & 102 & $0.92(1.25)$ & 185 & $66(36)$ & 76 & $0.59(1.00)$ & $1.56(1.11$ to 2.19$)$ \\
\hline $\begin{array}{l}\text { High AAP level } \\
(n=264)\end{array}$ & 148 & $62(42)$ & 95 & $0.74(1.13)$ & 113 & $58(51)$ & 116 & $1.26(2.15)$ & $0.61(0.42$ to 0.87$)$ \\
\hline \multicolumn{10}{|l|}{ Injurious falls } \\
\hline Overall & 299 & $121(40)$ & 224 & $0.75(1.47)$ & 298 & $130(44)$ & 235 & $0.79(1.25)$ & $0.96(0.74$ to 1.24$)$ \\
\hline Low AAP level & 151 & $65(43)$ & 138 & $0.91(1.84)$ & 185 & $77(42)$ & 126 & $0.68(1.02)$ & 1.34 (0.96 to 1.88$)$ \\
\hline High AAP level & 148 & $56(38)$ & 86 & $0.58(0.92)$ & 113 & $53(47)$ & 109 & $0.96(1.53)$ & 0.62 (0.42 to 0.92$)$ \\
\hline
\end{tabular}

Excludes nine participants with less than one month of follow-up.

AAP=Adelaide activities profile; low<median subtotal of 15 for outside activities; high $\geq$ median subtotal of 15 for outside activities.

*Number and percentage of participants who fell at least once in follow-up period.

tFalls per person over 13 month follow-up period. 
Table $3 \mid$ Secondary outcome variables at baseline and 13 month re-test. Values are means (SDs)

\begin{tabular}{|c|c|c|c|c|c|}
\hline \multirow[b]{2}{*}{ Measure } & \multicolumn{2}{|c|}{ Intervention } & \multicolumn{2}{|c|}{ Control } & \multirow[b]{2}{*}{$P$ value } \\
\hline & Pre-test & Re-test & Pre-test & Re-test & \\
\hline Falls efficacy scale_-international & $26.7(8.1)$ & $27.2(9.4)$ & $28.1(9.4)$ & $28.2(9.8)$ & 0.27 \\
\hline Adelaide activities profile & $36.9(9.6)$ & $35.1(8.8)$ & $34.1(8.8)$ & $36.5(8.9)$ & 0.17 \\
\hline SF-12 physical component score & $44.3(9.1)$ & $43.4(9.2)$ & $43.4(9.4)$ & $43.0(9.7)$ & 0.79 \\
\hline SF-12 mental component score & $54.3(6.6)$ & $53.6(7.4)$ & $54.5(6.7)$ & $54.1(5.6)$ & 0.47 \\
\hline
\end{tabular}

scores less than or equal to the median, intervention group participants had a non-significant increase in all falls and injurious falls and a significant increase in falls outside the home; the number needed to harm was 4.5. Sixteen (5\%) intervention group participants had one or more fall related fractures compared with 10 $(3 \%)$ control participants $\left(\chi^{2}=1.43, \mathrm{df}=1, \mathrm{P}=0.23\right)$. In the intervention group, fall rates did not differ significantly among participants categorised into quarters of self reported adherence $(\mathrm{P}=0.60)$.

Regardless of outdoor Adelaide activities profile subtotals, we found no significant differences between the two groups in fall related usage of general practitioner $(\mathrm{P}=0.15)$, outpatient specialist $(\mathrm{P}=0.83)$, emergency department $(\mathrm{P}=0.66)$, or inpatient hospital resources $(\mathrm{P}=0.63)$.

\section{Secondary outcome measures}

Table 3 shows the baseline and 13 month re-test scores for physical activity levels, falls efficacy, and quality of life measures for the intervention and control groups. We found no statistically significant between group differences for any of the secondary outcome measures at the end of the intervention after adjustment for baseline scores. No significant differences were evident when we did analyses relating to the secondary outcome measures for the Adelaide activities profile subgroups.

\section{Adverse events}

Two intervention participants and one control participant reported a fall while switching glasses. No intervention participants reported wearing single lens distance glasses when they had a fall related fracture: one reported wearing reading glasses, 12 multifocal glasses, and three no glasses; in two cases what type of glasses were worn, if any, was not clear. In the control group, nine participants reported wearing multifocal glasses and two reported wearing no glasses when a fall related fracture occurred; in one case what type of glasses were worn, if any, was unclear. Seventyseven $(26 \%)$ intervention participants had one or more non-fall injuries (laceration, lifting or twisting injury, burn/scald, eye injury, collision, pedestrian injuries) compared with $51(17 \%)$ controls $\left(\chi^{2}=6.61, \mathrm{df}=1\right.$, $\mathrm{P}=0.01)$.

\section{DISCUSSION}

With appropriate counselling, provision of single lens glasses for older wearers of multifocal glasses who take part in regular outdoor activities is an effective falls prevention strategy. Multifocal glasses have been shown to impair balance and increase the risk of falls in several studies. ${ }^{4-9}$ The blanket recommendation to avoid wearing them when walking would also often remove the correction of distance refractive error and subsequently increase the risk of falls in many older people. ${ }^{3}$ Thus, the fall prevention strategy used in this study was to supplement the use of multifocal glasses with an additional pair of single lens distance glasses for wearing when in outdoor and unfamiliar settings.

Intervention effect in relation to level of outdoor activity We found that total falls in the intervention group were reduced by $8 \%$ compared with the control group. Although this study was powered to detect a reduction in total falls, the lack of statistical significance is perhaps not surprising given that the intervention focused on outdoor activities and was therefore unlikely to have reduced indoor falls ( $47 \%$ of all falls). Also, the intervention had generally opposite effects on different subgroups according to Adelaide activities profile outdoor activity subtotals. Consequently, all falls, falls outside the home, and injurious falls were significantly reduced in intervention group participants who often left the home, a finding consistent with the rationale of the study.

In participants who left their homes less often, outside falls increased significantly. This is consistent with the findings of Cumming et $\mathrm{al}^{11}$; although no interactions existed between the intervention and the physiological fall risk score or previous falls, this may indicate that more cloistered (perhaps frailer) older people were less likely to benefit from a predominantly outdoor intervention and found the complication of adapting to additional glasses confusing. That the intervention generated false confidence and risk taking is also possible. However, we found no indication that falls occurred at the time of switching between multifocal and single lens distance glasses. The intervention did not influence the secondary outcome measures, indicating that the intervention did not increase physical activity or improve quality of life through fewer falls.

\section{Adherence and adverse events}

Adherence to the intervention was reasonable, particularly for an intervention that is less convenient than usual practice - that is, wearing a single pair of glasses. We suggest that this adequate adherence would not have been achieved without the assessment of self efficacy for the use of two pairs of glasses before 


\section{WHAT IS ALREADY KNOWN ON THIS TOPIC}

Multifocal glasses impair avoidance of obstacles and increase the risk of falls in older people

\section{WHAT THIS STUDY ADDS}

Provision of single lens glasses significantly reduced falls in older wearers of multifocal glasses who take part in regular outside activities

Older people who infrequently take part in regular outdoor activities may not benefit from this intervention

An increase in non-fall related injuries was associated with the intervention

randomisation and the initial counselling session. The counselling session took place in line with the health belief model, ${ }^{19}$ and it was important for convincing older wearers of multifocal glasses that they were personally at risk of fall related injuries and that the benefits of periodically switching to single lens glasses outweighed the inconvenience. Follow-up phone calls and follow-up sessions with the optometrist were provided for some intervention group participants who reported poor early adherence, but routine follow-up sessions may have been of value in further enhancing adherence.

Intervention group participants were more likely to have non-fall injuries. However, these injuries were generally minor and not related to increased healthcare use. This increased incidence of non-fall injuries seems to be related to a range of factors including the location of the incident and glasses worn at the time of the injury; we will report on this in detail in a separate paper. This finding highlights the potential for unintended consequences after any alteration to eyewear in older people either through a direct hazard or altered risk behaviour.

\section{Strengths and limitations of study}

The strengths of our study are its broad generalisability, high diary completion rates, small non-differential losses to follow-up, realistic adherence assumptions, and plausible pre-planned subgroup analyses. We acknowledge, however, that the study has certain limitations. As outlined in the flow diagram (figure), 357 people declined participation after initially expressing an interest in taking part for reasons such as that they realised that participation did not necessarily result in provision of free glasses, they considered 12 months' follow-up too long to commit to, or they thought that switching between two pairs of glasses needed too much effort. This would suggest a bias towards volunteers with heightened interest (and commitment) to the intervention. We were also unable to provide new glasses to all intervention group participants within the planned one month of randomisation, which may have caused an underestimation of the effect of the intervention. Thirdly, many of our results rely on the subgroup analysis using Adelaide activities profile outdoor activity items. The validity of this assessment is supported by separate analyses, which showed that Adelaide activities profile subtotals correlated with amount of self reported outdoor walking $(\mathrm{P}<0.001)$. Fourthly, we did not ascertain to what extent transferring the cost of purchasing additional glasses would reduce adoption of this intervention. Finally, we acknowledge that, as the single lens glasses provided to the intervention group participants were tinted, the resulting improved contrast sensitivity may have been an additional factor in the reduction in falls, especially for people with early cataracts and in the Australian sun. $^{20}$

\section{Policy and practice implications}

On the basis of our findings and previous publications, ${ }^{48911}$ the following recommendations for the prescription of multifocal glasses for older people can be made. Firstly, for older people who have minimal correctable distance refractive error, multifocal glasses should be avoided. This group need only reading glasses for near vision that can be removed when walking. Current owners of multifocal glasses with minimal correctable distance refractive error should also remove these glasses when walking outdoors. Secondly, older people with considerable correctable distance refractive error who take part in frequent outdoor activities should use single lens distance glasses when outdoors and in other unfamiliar settings. To assist this, single lens glasses should be provided for outside use when a person is prescribed his or her first pair of multifocal glasses. Thirdly, older regular wearers of multifocal glasses with considerable correctable distance refractive error who take part in little outdoor activity should use multifocal glasses for most activities (rather than using multiple pairs of glasses). Fourthly, in terms of fall risk, insufficient evidence exists to recommend one type of multifocal glasses over another. Finally, any change in prescription for distance components of either multifocal or single vision glasses should be conservative. ${ }^{11}$

\section{Conclusions}

These findings suggest that, with appropriate assessment and counselling, the provision of single lens glasses for older people who take part in regular outdoor activities is a simple and effective falls prevention strategy. However, given that our study and the trial by Cumming et al showed increases in outdoor falls in some participants, clinicians should be conservative in eyewear prescription, particularly in frail older people who do not often leave their homes. ${ }^{11}$

The VISIBLE study team thanks the following people and organisations for their assistance with the project: M Ding, J Evans, G Matthews, G Bigland, M Michael, J Sleeman, J Comber, and D Semmonds for visual assessments; J Jowitt, S Mount, W Rogan, L Fine, T Thurn, O Marial,

X Masgoret, P Edwards, J Fullarton, L Lee, O Lee, M Lee, M Luig, R Haran, J Close, R Hinchey, P McDermott, J Zheng, Dee Why and Chester Hill

Probus Clubs, Chatswood Day Centre, War Veterans Village Collaroy, and Jean Waldren for logistical support and technical advice; R Lochlin,

M Armstrong, C Angstman, L Holliday, K Tattersall, C Swinton, A Haidary, A Collins, D Wilton, F Scott, R Carroll, N Rich, J Prior, E Orchiston, D Taylor, I Ford, M Roth, D Lewis, D Taylor, and S Wise for study recruitment; and all participants and recruitment centres.

Contributors: SRL and MJH were involved in study concept and design, study coordination, and data analysis and interpretation. IDC, RQI, JMS, 
and BBL were involved in study concept and design and data interpretation. MT was responsible for optometry assessment and counselling. MP and MMSK were responsible for participant recruitment, database management, and administration of questionnaires and physical tests. CS provided study coordination assistance and was involved in database management. All authors were involved in preparation of the manuscript. SRL and MJH are the guarantors. Funding: This study was funded by an Australian National Health and Medical Research Council grant (reference number NHMRC ID 350855) in 2005. The research was conducted independently from the funding body.

Competing interests: The physiological profile assessment (FallScreen) is commercially available through the Prince of Wales Medical Research Institute.

Ethical approval: The University of New South Wales Human Research Ethics Committee approved the study protocol (15/02/2005). All participants gave informed consent.

Data sharing: no additional data available.

1 Donahue SP. Loss of accommodation and presbyopia. In: Yanoff M, Duker JS, eds. Ophthalmology. Mosby, 1999.

2 Duke Elder S. The practice of refraction. Churchill, 1963.

3 Tinetti ME. Clinical practice: preventing falls in elderly persons. $N$ Engl J Med 2003;348:42-9.

4 Lord SR, Dayhew J, Howland A. Multifocal glasses impair edge contrast sensitivity and depth perception and increase the risk of falls in older people. J Am Geriatr Soc 2002;50:1760-6.

5 Johnson L, Buckley JG, Harley C, Elliott DB. Use of single-vision eyeglasses improves stepping precision and safety when elderly habitual multifocal wearers negotiate a raised surface. J Am Geriatr Soc 2008;56:178-80.

6 Johnson L, Buckley JG, Scally AJ, Elliott DB. Multifocal spectacles increase variability in toe clearance and risk of tripping in the elderly. Invest Ophthalmol Vis Sci 2007;48:1466-71.

7 Menant JC, St George RJ, Sandery B, Fitzpatrick RC, Lord SR. Older people contact more obstacles when wearing multifocal glasses and performing a secondary visual task. J Am Geriatr Soc 2009;57:1833-8.
8 Connell BR, Wolf SL. Environmental and behavioral circumstances associated with falls at home among healthy elderly individuals. Arch Phys Med Rehabil 1997;78:179-86.

9 Davies JC, Kemp GJ, Stevens G, Frostick SP, Manning DP. Bifocal/ varifocal spectacles, lighting and missed-step accidents. Safety $\mathrm{Sc}$ 2001;38:211.

10 Day L, Fildes B, Gordon I, Fitzharris M, Flamer H, Lord S. Randomised factorial trial of falls prevention among older people living in their own homes. BMJ 2002;325:128-31.

11 Cumming RG, Ivers R, Clemson L, Cullen J, Hayes MF, Tanzer M, et al. Improving vision to prevent falls in frail older people: a randomized trial. J Am Geriatr Soc 2007;55:175-81.

12 Haran MJ, Cameron ID, Ivers RQ, Simpson JM, Lee BB, Porwal M, et al. Preventing falls in older multifocal glasses wearers by providing single-lens distance glasses: the protocol for the VISIBLE randomised controlled trial. BMC Geriatrics 2009;9:10.

13 Gibson MJ, Andres RO, Isaacs B, Radebaugh T, Worm-Petersen J. The prevention of falls in later life. Dan Med Bull 1987;34(suppl 4):1-24S.

14 Clark MS, Bond MJ. The Adelaide activities profile: a measure of the lifestyle activities of elderly people. Aging Clin Exp Res 1995;7:174-84.

15 Gandek B, Ware J, Aaronson NK, Apolone G, Bjorner JB, Bullinger M, et al. Cross-validation of item selection and scoring for the SF-12 health survey in nine countries: results from the IQOLA project. J Clin Epidemiol 1998;51:1171-8.

16 Yardley L, Beyer N, Hauer K, Kempen G, Piot-Ziegler C, Todd C. Development and initial validation of the falls efficacy scale international (FES-I). Age Ageing 2005;34:614-9.

17 Brookes ST, Whitely E, Egger M, Smith GD, Mulheran PA, Peters TJ. Subgroup analyses in randomized trials: risks of subgroup-specific analyses; power and sample size for the interaction test. J Clinical Epidemiol 2004;57:229-36.

18 Lord SR, Menz HB, Tiedemann A. A physiological profile approach to falls risk assessment and prevention. Phys Ther 2003;83:237-52.

19 Rosenstock I. The health belief model and preventive health behaviour. Health Educ Monogr 1978;2:354-86.

20 Naidu S, Lee JE, Holopigian K, Seiple WH, Greenstein VC, Stenson SM. The effect of variably tinted spectacle lenses on visual performance in cataract subjects. Eye Contact Lens 2003;29:17-20.

Accepted: 11 April 2010 